Proyecciones

Vol. 18, $\mathrm{N}^{\circ}$ 1, pp. 127-136, July 1999

Universidad Católica del Norte

Antofagasta - Chile

\title{
Sur l'homologie des déformations de modules différentiels
}

\author{
ALAIN GUICHARDET \\ Centre de Mathématiques, École Polytechnique, France
}

\begin{abstract}
Résumé. Ce travail est consacré à la démonstration de deux résultats concernant l'homologie des déformations de modules différentiels, et reliant respectivement l'homologie d'une déformation formelle $\grave{a}$ celle d'une déformation "à paramètre fixé" et à celle de la déformation triviale.
\end{abstract}

Summary. This work is devoted to the proof of two results concerning the homology of deformations of differential modules, and relating respectively the homology of a formal deformation to the homology of a deformation "with fixed parameter" and of the trivial deformation. 


\section{Introduction}

Dans l'étude des déformations de structures algébriques, on peut adopter deux points de vue apparemment assez distincts : celui des "déformations formelles" et celui dit "à paramètrc fixé". Pronons par exemple le plan quantique; on part de l'algèbre $A$ des polynômes à deux indéterminées sur un corps $k ; A$ est engendrée par deux éléments $X_{1}$ et $X_{2}$ soumis à la relation $X_{1} X_{2}=X_{2} X_{1}$; on peut considérer deux sortes de déformations de $A$ :

1) une algèbre $A_{h}$ sur l'anneau $k:[[h]]$ des sćrics formelles à coefficients dans $k ; A_{h}$ est engendrée par les élénents $X_{1}$ et $X_{2}$ soumis à la relation $X_{1} X_{2}=e^{h} X_{2} X_{1}$ où $e^{h}$ est la sćric formelle $\sum_{n \geq 0} h^{n} / n !$;

2) une algèbre $A_{q}$ sur $k$, où maintenant $q$ est un ćlémont fixé non nul de $k ; A_{q}$ est engendrée par les éléments $X_{1}$ et $X_{2}$ soumis à la relation $X_{1} X_{2}=q X_{2} X_{1}$.

On constate que, si $q$ est suffisamment "générique" (par exemple non racine de l'unité), les algèbres $A_{h}$ et. $A_{q}$ ont des propriétés très semblables, notamment en ce qui concerne l'homologie ou la cohomologic de Hochsschild. Mieux, certains auteurs, étudiant le point de vue "à paramètre fixé", passent au point de vue "déformation formelle" pour pouvoir utiliser une suite spectrale associée à la filtration d'un certain complexe par les puissances de $h$ (cf. [4], [6]).

L'objet de la première partie du présent travail est d'énoncer un résultat. à la fois précis et général reliant les homologies de deux modules diffórentiels respectivement sur $k$ et $k[[h]]$; sa démonstration repose sur des propriétés classiques, mais non triviales, des modules plats et des modules sur des anneaux principaux.

Dans la seconde partie, on donne une condition suffisante pour que l'homologie d'un module différentiel $K$ sur $k[[h]]$ se calcule simplement à partir de l'homologie du $k$-module différenticl $K / h K$; ce résultat peut se déduire des propriétés générales des suites spectrales, mais il m'a semblé utile d'en donner une preuve directe et élémentaire. 


\section{Relation entre les points de vue "déformations}

formelles" et "à paramètre fixé".

Notations. On désignera par

- $k$ un corps commutatif de caractéristique 0 ;

- $k^{\prime}$ l'anneau de séries formelles $k[[h]]$;

- $q$ un élément de $k$ transcendant sur le corps $Q$ des rationnels;

- $\lambda$ un élément de $k^{\prime}$ transcendant sur $Q$;

- $C$ un $k$-espace vectoriel de dimension finie notée $m$, muni d'une base $\left(e_{i}\right)$;

- $P_{i, j}, i, j=1, \ldots, m$ des polynômes à une indéterminée sur $Q$ vérifiant $\sum_{j} P_{i, j} P_{j, k}=0$ pour tout $i$ et tout $k$;

- $d$ le $k$-endomorphisme de $C$ de coefficients $d_{i, j}=P_{i, j}(q)$; on a $d^{2}=0$;

- $C^{\prime}$ le $k^{\prime}$-module $C \otimes_{k} k^{\prime}=C[[h]]$;

- $d^{\prime}$ le $k^{\prime}$-endomorphisme de $C^{\prime}$ de coefficients $d_{i, j}=P_{i, j}(\lambda)$; on a $d^{\prime 2}=0$;

- $H(C, d)$ l'homologie du module différentiel $(C, d)$, i.e. $H(C, d)=\operatorname{Ker} d / \operatorname{Im} d$;

- $H\left(C^{\prime}, d^{\prime}\right)$ l'homologie du module différentiel $\left(C^{\prime}, d^{\prime}\right)$.

On rappelle que $k^{\prime}$ est un anneau principal : tout idéal de $k^{\prime}$ est engendré par une puissance de $h$; si $X$ est un $k^{\prime}$-module, on désigne par $X^{t}$ son sousmodule de torsion, ensemble des éléments anmulés par une puissance de $h$ , et on pose $X^{s t}=X / X^{t}$ (quotient sans torsion de $X$ ).

Théorème 2.1. Il existe un isomorphisme de k'-modules

$$
H\left(C^{\prime}, d^{\prime}\right)^{s t} \approx H(C, d) \otimes_{k} k^{\prime} .
$$

Autres notations. On notera $k^{\prime \prime}$ le corps des quotients de $k^{\prime}$ et on posera

$C^{\prime \prime}=C^{\prime} \otimes_{k^{\prime}} k^{\prime \prime}=C \otimes_{k} k^{\prime \prime}$

$d^{\prime \prime}=d^{\prime} \otimes_{k^{\prime}} i d_{k^{\prime \prime}}\left(k^{\prime \prime}\right.$-endomorphisme de $C^{\prime \prime}$ de coefficients $\left.P_{i, j}(\lambda)\right)$

$d^{\prime \prime \prime}=d \otimes_{k} i d_{k^{\prime \prime}}\left(k^{\prime \prime}\right.$-endomorphisne de $C^{\prime \prime}$ de coefficients $\left.P_{i, j}(q)\right)$.

Lemme 2.1. On a $H\left(C^{\prime \prime}, d^{\prime \prime}\right) \approx H\left(C^{\prime}, d^{\prime}\right) \otimes_{k^{\prime}} k^{\prime \prime}$.

Démonstration. Le corps $k^{\prime \prime}$ est un $k^{\prime}$-module plat (cf.[1], ch. II, §2, théorème 1) et cela implique notre assertion ([1], ch I, $\S 2, N^{o} 3$, définition 2 et remarque 2 qui suit). 
Lemme 2.2. On a $H\left(C^{\prime \prime}, d^{\prime \prime \prime}\right) \approx H(C, d) \otimes_{k} k^{\prime \prime}$.

Démonstration. Même démonstration qu'au lemme 2.1, puisque $k^{\prime \prime}$ est un $k$-module plat.

Lemme 2.3. On a $H\left(C^{\prime \prime}, d^{\prime \prime}\right) \approx H\left(C^{\prime \prime}, d^{\prime \prime \prime}\right)$.

Cela résulte du résultat plus général suivant:

Lemme 2.4. Soit $K$ un corps de caractóristique nulle, $q_{1}$ et $q_{2}$ des éléments de $K$ transcendants sur $Q, E$ un $K$-cspace vectoricl de dinension finie muni d'une base $\left(e_{i}\right),\left(P_{i, j}\right)$ des polynômes ayant les propriétés indiquées ci-dessus, enfin $d_{1}$. (resp. $\left.d_{2}\right)$ le $K$-endomorplisme de $E$ de cocfficionts $P_{i, j}\left(d_{1}\right)$ (resp. $P_{i, j}\left(d_{2}\right)$ ). Alors

$$
H\left(E, d_{1}\right) \approx H\left(E, d_{2}\right)
$$

Démonstration. Pour $n=1,2$, notons $K_{n}$ le sous-corps de $K$ engendré par $q_{n}$ ct $E_{n}$ le sous- $K_{n}$-espace vectoricl de $E$ engendré par les $e_{i} ; E_{n}$ est stable par $d_{n}$ et $\left(E, d_{n}\right)$ s'obticnt par extension des scalaires à partir de $\left(E_{n}, d_{n}\right)$; ceci implique, conme au lemme 2.2 , que

$$
H\left(E, d_{n l}\right) \approx H\left(E_{n}, d_{n}\right) \otimes_{K_{n}} K .
$$

Par ailleurs $K_{1}$ ct $K_{2}$ sont isomorphes au corps de fractions rationnelles $Q(X), E_{1}$ ct $E_{2}$ s'identifient au $Q(X)$-espace vectoricl $F$ de base $\left(e_{i}\right)$, ct $d_{1}$ et $d_{2}$ s'identifient au $Q(X)$-cndomorphismo de $F$ de cocfficients $P_{i, j}(X)$.

Lemme 2.5. On a

$$
H\left(C^{\prime}, d^{\prime}\right) \otimes_{k^{\prime}} k^{\prime \prime} \approx H\left(C^{\prime}, d^{\prime}\right)^{s t} \otimes_{k^{\prime}} k^{\prime \prime} .
$$

Démonstration. Notons pour simplificr $M$ le $k^{\prime}$-modulc $H\left(C^{\prime}, d^{\prime}\right)$ et $M^{t}$ son sous-module de torsion. Comme $k^{\prime \prime}$ est un $k^{\prime}$-module plat, la suite exactc

$$
0 \rightarrow M^{t} \rightarrow M \rightarrow M^{s t} \rightarrow 0
$$

entraîno une suite exacte

$$
0 \rightarrow M^{t} \otimes_{k^{\prime}} k^{\prime \prime} \rightarrow M \otimes_{k^{\prime}} k^{\prime \prime} \rightarrow M^{s t} \otimes_{k^{\prime}} k^{\prime \prime} \rightarrow 0 ;
$$


mais $M^{t} \otimes_{k^{\prime}} k^{\prime \prime}$ est nul ; en effet, pour tout élément $m \otimes \mu$ de $M^{t} \otimes_{k^{\prime}} k^{\prime \prime}$, il existe $\lambda$ non nul dans $k^{\prime}$ tel que $\lambda m=0 ; \lambda$ est inversible dans $k^{\prime \prime}$ (qui est un corps), donc

$$
m \otimes \mu=m \otimes \lambda \lambda^{-1} \mu=\lambda m \otimes \lambda^{-1} \mu=0 .
$$

Démonstration du théorème. Les lemmes $2.1,2.2,2.3$ et 2.5 montrent que

$$
H\left(C^{\prime}, d^{\prime}\right)^{s t} \otimes_{k^{\prime}} k^{\prime \prime} \approx H(C, d) \otimes_{k} k^{\prime \prime}
$$

ou encore

$$
H\left(C^{\prime}, d^{\prime}\right)^{s t} \otimes_{k^{\prime}} k^{\prime \prime} \approx\left(H(C, d) \otimes_{k} k^{\prime}\right) \otimes_{k^{\prime}} k^{\prime \prime} .
$$

Le $k^{\prime}$-module $C^{\prime}$ est libre de rang fini ; comme $k^{\prime}$ est un anneau principal, le sous- $k^{\prime}$-module Ker $d^{\prime}$ est aussi libre de rang fini (cf. [2], ch. VII, §3, prop. 1) ; $\mathrm{H}\left(\mathrm{C}^{\prime}, \mathrm{d}^{\prime}\right)$, quotient de Ker d', est de type fini ; $H\left(C^{\prime}, d^{\prime}\right)^{\text {st }}$ est de type fini et sans torsion, donc libre de rang fini (cf. [2], ch. VII, $\S 4, \mathrm{~N}^{\circ}$ 3 , cor. 2). Le $k^{\prime}$-module $H(C, d) \otimes_{k} k^{\prime}$ est évidemment aussi libre de rang fini ; alors (1) montre que les rangs de $H\left(C^{\prime}, d^{\prime}\right)^{s t}$ et $H(C, d) \otimes_{k} k^{\prime}$ sont tous deux égaux à la dimension du $k^{\prime \prime}$-espace vectoriel $H\left(C^{\prime}, d^{\prime}\right)^{s t} \otimes_{k^{\prime}} k^{\prime \prime}$.

Exemple. (Homologie de Hochschild de l'espace affine quantique de dimension $N$; cf. [5]).

On part de l'algèbre de polynômes $A=k\left[X_{1}, \ldots, X_{N}\right]$ et on considère

1) une déformation formclle $A_{h}$ de $A$, algèbre sur $k^{\prime}$ engendrée par les éléments $X_{1}, \ldots, X_{N}$ soumis aux relations

$$
X_{i} X_{j}=e^{h} X_{j} X_{i} \quad \text { pour } i<j ;
$$

2) une déformation "à paramètre fixé" $A_{q}$ de $A$, algèbre sur $k$ engendrée par les éléments $X_{1}, \ldots, X_{N}$ soumis aux relations

$$
X_{i} X_{j}=q X_{j} X_{i} \quad \text { pour } i<j .
$$

Dans les deux cas, le complexe qui calcule l'homologie de Hochschild est de dimension infinie, mais on se ramène à des dimensions finies en se limitant aux éléments homogènes de degré fixé ; le théorème 2.1 montre alors que, si $q$ est transcendant sur $Q$,

$$
H H_{n}\left(A_{h}\right)^{s t} \approx H H_{n}\left(A_{q}\right) \otimes_{k} k^{\prime} .
$$


Notons que $H H_{n}\left(A_{h}\right)$ n'est pas sans torsion en général ; par exemple $H H_{0}\left(A_{h}\right)$ est le quotient de $A_{h}$ par le sous-module $\left[A_{h}, A_{h}\right]$ engendré par les commutateurs ; lorsque $N=2$, on peut écrire les éléments de $A_{h}$ sous la forme

$$
a=\sum_{m \geq 0} \sum_{n \geq 0} a_{m, n} X_{1}^{m} X_{2}^{n}
$$

alors $\left[A_{h}, A_{h}\right]$ est l'ensemble des éléments de $A_{h}$ de la forme $h a$ avec

$$
a=\sum_{m>0} \sum_{n>0} a_{m, n} X_{1}^{m} X_{2}^{n}
$$

on voit que $h X_{1} X_{2}$ appartient à $\left[A_{h}, A_{h}\right]$, mais pas $X_{1} X_{2}$.

Remarque. On peut généraliser le théorène 1 en prenant $n$ éléments $q_{1}, \ldots, q_{n}$ (resp. $\lambda_{1}, \ldots, \lambda_{n}$ ) algébriquement indépendants de $k$ (resp. $k^{\prime}$ ) et des polynômes $P_{i, j}$ à $n$ indéterminées. Cela s'applique notamment à l'homologie de Hochschild de l'algèbre engendrée par 3 éléments $X_{1}, X_{2}$, $X_{3}$ soumis aux relations

$$
X_{1} X_{2}=e^{h} X_{2} X_{1}, X_{2} X_{3}=e^{h} X_{3} X_{2}, X_{1} X_{3}=X_{3} X_{1}+h X_{2}^{2} ;
$$

on prendra ici $n=2, \lambda_{1}=h, \lambda_{2}=e^{h}$ (cf. [5]).

\section{Passage à la limite classique.}

\section{Notations.}

On défnit $k$ et $k^{\prime}$ comme au $\S 2$; on désigne par $K_{0}$ un $k$-espace vectoriel de dimension finie et par $d$ un $k^{\prime}$-endomorphisme de carré nul de $K=K_{0} \otimes_{k} k^{\prime}$; on identifie $K / h K$ avec $K_{0}$ et on note $d_{0}$ le $k$-endomorphisme de $K_{0}$ obtenu à partir de $d$ par passage au quotient. L'application canonique $K \rightarrow K_{0}$ entraîne une application $\Phi: H(K, d) \rightarrow H\left(K_{0}, d_{0}\right)$.

Théorème 3.1. Si $\Phi$ est surjective, il cxiste des isomorphismes de $k^{\prime}$ modules

$$
H(K, h d)^{s t} \approx H(K, d) \approx H\left(K_{0}, d_{0}\right) \otimes_{k} k^{\prime}
$$


Notations. Pour tout $k \in Z$ on pose $K^{p}=h^{p} K$ (égal à $K$ si $p \leq 0$ ) ; on a $d\left(K^{p}\right) \subset K^{p}$ et on note $d^{p}$ la restriction de $d$ à $K^{p}$. On pose $G^{p}=K^{p} / K^{p+1}$; on désigne par $\pi^{p}$ l'application canonique $K^{p} \rightarrow G^{p}$, par $\partial^{p}$ l'application $G^{p} \rightarrow G^{p}$ obtenue à partir de $d^{p}$ par passage au quotient, et par $\lambda^{p}$ l'application $H\left(K^{p}, d^{p}\right) \rightarrow H\left(G^{p}, \partial^{p}\right)$ déduite de $\pi^{p}$. Pour $p=0$, on a $K^{0}=K, G^{0}=K_{0}, \lambda^{0}=\Phi$.

Lemme 3.1. L'application $\lambda^{p}$ est surjective pour tout $p$.

Démonstration. On a des isomorphismes $K^{0} \rightarrow K^{p}\left(x \rightarrow h^{p} x\right)$ et $G^{0} \rightarrow$ $G^{p}$ qui transforment $\lambda^{0}$ en $\lambda^{p}$.

Lemme 3.2. On a

$$
K^{p} \cap d^{-1}\left(K^{p+1}\right) \subset \operatorname{Ker} d^{p}+K^{p+1} .
$$

Démonstration. Comme $\lambda^{p}$ est surjectif, on a

Ker $\partial^{p}=\operatorname{Im} \partial^{p}+\pi^{p}\left(\operatorname{Ker} d^{p}\right)=\pi^{p}\left(\operatorname{In} d^{p}\right)+\pi^{p}\left(\operatorname{Ker} d^{p}\right)=\pi^{p}\left(\operatorname{Ker} d^{p}\right) ;$

par ailleurs on a facilement $K^{p} \cap d^{-1}\left(K^{p+1}\right) \subset\left(\pi^{p}\right)^{-1}\left(K e r \partial^{p}\right)$ d'où le résultat.

Lemme 3.3. On a

$$
K^{p} \cap d(K) \subset d\left(K^{p}\right)+K^{p+1} .
$$

Démonstration. Pour tout $r=0,1, \ldots, p$ posons $L^{r}=K^{p} \cap d\left(K^{p-r}\right)$; on a évidemment

$$
L^{0} \subset L^{1} \subset \ldots \subset L^{p-1} \subset L^{p}=K^{p} \cap d(K)
$$

et

$$
L^{0}=K^{p} \cap d\left(K^{p}\right) \subset d\left(K^{p}\right)+K^{p+1} .
$$


On va montrer que, pour $1 \leq r \leq p$, on a $L^{r}=L^{r-1}$. Posons $q=p-r$; soit $x \in L^{r}=K^{q+r} \cap d\left(K^{q}\right) ; x$ s'écrit $x=d y$ avec $y \in K^{q} \cap d^{-1}\left(K^{q+r}\right) \subset$ $K^{q} \cap d^{-1}\left(K^{q+1}\right)$; le lemme 3.2 montre que $y \in K e r d^{q}+K^{q+1}$, et cela implique immédiatement $x \in L^{r-1}$.

Lemme 3.4. Notons $\chi$ l'application canonique $\mathrm{Kor} d \rightarrow H(K, d)$, $\chi^{p}$ sa restriction à Ker d $d^{p}$ et posons

$$
H(K, d)^{p}=\operatorname{Im} \chi^{p}=\chi\left(\operatorname{Ker} d^{p}\right) .
$$

On a des isomorphismes

$$
H(K, d)^{p} / H(K, d)^{p+1} \approx H\left(G^{p}, \partial^{p}\right) .
$$

\section{Démonstration.}

Soit $x \in \operatorname{Ker} d^{p}$; le lemme 3.3 montre que, si $\chi^{p}(x)=0, \pi^{p}(x) \in \operatorname{Im} \partial^{p}$; on a clonc unc application $\mu^{p}: H(K, d)^{p} \rightarrow H\left(G^{p}, \partial^{p}\right)$, qui est surjective d'après le lemme 3.1. Roste à voir que l'on a Kor $\mu \iota^{p}=H(K, d)^{p+1}$. L'inclusion $H(K, d)^{p+1} \subset K$ cr $\mu^{p}$ ćtant facile à vérificr, démontrons l'autre. Soit $y \in \operatorname{Ker} \mu^{p}, x \in \operatorname{Ker} d^{p}$ tel que $y=\chi^{p}(x) ;$ on a $\pi^{p}(x) \in \operatorname{Im} \partial^{p}=$ $\pi^{p}\left(d\left(K^{-p}\right)\right) ; x$ s'écrit $d u+v$ avec $u \in K^{-p}$ et $v \in K^{p+1}$; on a $d v=d x=0$, dons: $v \in K e r d^{p+1}$; d'où $y=\chi(v) \in H(K, d)^{p+1}$.

\section{Démonstration du théorème 3.1 .}

a) Démontrons que $H(K, d) \approx H\left(\kappa_{0}, d_{0}\right) \otimes_{k} k^{\prime}$. Comme les modules différentiels $\left(G^{p}, \partial^{p}\right)$ et $\left(K_{0}, d_{0}\right)$ sont isomorphes, lo lemme 3.4 montre que l'on a

$$
H(K, d)^{p} / H(K, d)^{p+1} \approx H\left(K_{0}, d_{0}\right) .
$$

Par ailleurs il suffit de montrer que $H(K, d)$ est un $k^{\prime}$-module libre de rang fini. On a vu à la démonstration du théorème 2.1 que $H(K, d)$ est de type fini ; d'après [2], ch. VII, $\S 4, N^{o} 3$, cor.1. $H(K, d)$ est somme directe d'un module libre de rang fini $X$ et d'un module de torsion de type fini $Y$; il existe done un entier $n \geq 0$ tel que $h^{n} Y=0$. D'autre part on a

$$
H(K, d)^{p}=h^{p} H(K, d)=h^{p} X \oplus h^{p} Y
$$

la relation (1) entraîne

$$
H\left(K_{0}, d_{0}\right)=\left(h^{p} X / h^{p+1} X\right) \oplus\left(h^{p} Y / h^{p+1} Y\right)=(X / h X) \oplus\left(h^{p} Y / h^{p+1} Y\right) ;
$$


ceci montre que $h^{p} Y / h^{p+1} Y$ est indépendant de $p$; comme il est nul pour $p=n$, il est nul pour tout $p$, d'où

$$
Y=h Y=h^{2} Y=\cdots=h^{n} Y=0 ;
$$

$H(K, d)$ est libre de rang fini et (1) entraîne notre assertion.

b) Démontrons que $H(K, h d)^{s t} \approx H(K, d)$. Notons $u$ et $v$ les applications canoniques Ker $h d \rightarrow H(K, h d)$ et Ker $d(=\operatorname{Ker} h d) \rightarrow H(K, d)$ et $w$ l'application $H(K, h d) \rightarrow H(K, d)$ qui s'en déduit. On va montrer que $\operatorname{Ker} w=H(K, h d)^{t}$; si $x \in H(K, h d)^{t}$, il existe $n>0$ tel que $h^{n} x=0$; $x$ s'écrit $u(y)$ avec $y \in K e r d$ et $u\left(h^{n} y\right)=0$; on a alors $h^{n} y \in \operatorname{Im} h d \subset \operatorname{Im} d$; comme $H(K, d)$ est sans torsion, cela implique $y \in \operatorname{Im} d$, d'où $x \in \operatorname{Ker} w$. L'inclusion inverse est immédiate.

Remarque. On peut démontrer le lemme 3.4 plus rapidement en utilisant la théorie des suites spectrales ; On a vu que $\pi^{p}$ envoie $K^{p} \cap d^{-1}\left(K^{p+1}\right)$ dans Ker $\partial^{p}$; notons $\rho^{p}$ l'application $K^{p} \cap d^{-1}\left(K^{p+1}\right) \rightarrow H\left(G^{p}, \partial^{p}\right)$ obtenue par composition. La suite spectrale associée à la filtration de $K$ par les $K^{p}$ comporte les ensembles suivants (dans les notations de [3]) :

$$
\begin{aligned}
& Z_{1}^{p}=\rho^{p}\left(K^{p} \cap d^{-1}\left(K^{p+1}\right)\right) \\
& \left.Z_{\infty}^{p}=\rho^{p}\left(K^{p} \cap \operatorname{ker} d\right)\right) \\
& B_{\infty}^{p}=\rho^{p}\left(K^{p} \cap d(K)\right) .
\end{aligned}
$$

Le lemme 3.2 montre que $Z_{1}^{p}=Z_{\infty}^{p}$; les propriétés générales des suites spectrales montrent, d'une part, que cela implique $B_{\infty}^{p}=0$, et, d'autre part, que l'on a toujours

$$
Z_{1}^{p} \approx H\left(G^{p}, \partial^{p}\right) \quad, \quad Z_{\infty}^{p} / B_{\infty}^{p} \approx H(K, d)^{p} / H(K, d)^{p+1} .
$$

Application. Considérons une déformation formelle quadratique $A_{h}$ d'une algèbre de polynômes $k\left[x_{1}, \ldots, x_{N}\right]$ ayant les propriétés énumérées dans [5], No III.1 et supposons en outre que $R_{n}$ admet une base de la forme indiquée dans la proposition 2 de [5]; l'homologie de Hochschild $H H_{*}\left(A_{h}\right)$ de $A_{h}$ est l'homologie d'un complexe noté $\left(C_{*}, \partial_{*}\right)$ dans [5], No III.4; posons $\partial_{*}^{\prime}=h^{-1} \partial_{*}$ comme dans [5], §4. Pour appliquer le théorème 3.1 ci-dessus, on prend pour $(K, d)$ le sous-complexe de $\left(C_{*}, \partial_{*}^{\prime}\right)$ formé des éléments homogènes ayant un degré fixé ; d'après un résultat non publié de $J$. Pichaud, lc complexe $\left(K_{0}, d_{0}\right)$ est colui qui calcule l'homologie $H P_{*}\left(k^{N}, \Pi\right)$ de la structure de Poisson sur $k\left[x_{1}, \ldots, x_{N}\right]$ associée à la cléformation $A_{h}$. Le 
théorème 3.1 ci-dessus dit alors que, si l'application canonique $H\left(C_{*}, \partial_{*}^{\prime}\right) \rightarrow$ $H P_{*}\left(k^{N}, \Pi\right)$ est surjective, on a des isomorphismes

$$
H H_{*}\left(A_{h}\right)^{s t} \approx H\left(C_{*}, \partial_{*}^{\prime}\right) \approx H P_{*}\left(k^{N}, \Pi\right) \otimes_{k} k^{\prime} .
$$

L'intérêt de ce résultat tient au fait que le calcul de $H P_{*}\left(k^{N}, \Pi\right)$ est plus simple que celui de $H H_{*}\left(A_{h}\right)^{\text {st }}$; c'est d'ailleurs la méthode employée dans [6] pour faire un calcul de ce type.

\section{References}

[1] N. Bourbaki, Algèbre commutative.

[2] N. Bourbaki, Algèbre.

[3] H. Cartan-S.Eilenberg, Homological algebra, Princeton Univ. Press (1956).

[4] P. Feng-B.Tsygan, Hochschild and cyclic homology of quantum groups, Commun. Math. Phys., t. 140, pp. $481-521$, (1991)

[5] A. Guichardet, Homologie de Hochschild des déformations quadratiques d'algèbres de polynômes, Communications in Algebra, t. 26/12, pp. $4309-4330,(1998)$

[6] M. VAN DEN BERGH, Non commutative homology of some threedimensional quantum spaces, K-theory, t. 8, pp. 213 - 230, (1994)

Received : November, 1998

\section{Alain Guichardet}

Centre de Mathématiques

Ecole Polytechnique

URA 169 du C. N. R. S.

91.128 Palaiseau, Cedex

France 